\title{
Dynamic Power Allocation and Routing for Time Varying Wireless Networks
}

\author{
Michael J. Neely \\ http://web.mit.edu/mjneely/www \\ MIT_LIDS: mjneely@mit.edu
}

\author{
Eytan Modiano \\ http://web.mit.edu/modiano/www \\ MIT_LIDS: modiano@mit.edu
}

\author{
Charles E. Rohrs \\ MIT-LIDS \\ crohrs@mit.edu
}

\begin{abstract}
We consider dynamic routing and power allocation for a wireless network with time varying channels. The network consists of power constrained nodes which transmit over wireless links with adaptive transmission rates. Packets randomly enter the system at each node and wait in output queues to be transmitted through the network to their destinations. We establish the capacity region of all rate matrices $\left(\lambda_{i j}\right)$ that the system can stably support-where $\left(\lambda_{i j}\right)$ represents the rate of traffic originating at node $i$ and destined for node $j$. A joint routing and power allocation policy is developed which stabilizes the system and provides bounded average delay guarantees whenever the input rates are within this capacity region. Such performance holds for general arrival and channel state processes, even if these processes are unknown to the network controller. We then apply this control algorithm to an ad-hoc wireless network where channel variations are due to user mobility, and compare its performance with the Grossglauser-Tse relay model developed in [13].
\end{abstract}

\section{INTRODUCTION}

Wireless systems have emerged as a ubiquitous part of modern data communication networks. Demand for these systems continues to grow as applications involving both voice and data expand beyond their traditional wireline service requirements. In order to meet the increasing demand in data rates that are currently being supported by high speed wired networks composed of electrical cables and optical links, it is important to fully utilize the capacity available in wireless systems, as well as to develop robust strategies for integrating these systems into a large scale, heterogeneous data network. Emerging microprocessor technologies are enabling wireless units to be equipped with the processing power needed to implement adaptive coding techniques and to make intelligent decisions about packet routing and resource management. It is expedient to take full advantage of these capabilities by designing efficient network control algorithms.

In this paper, we develop algorithms for dynamic routing and power allocation in a wireless network consisting of $N$ power constrained nodes. Time is slotted, and every timeslot the channel conditions of each link randomly change (due to external effects such as fading, user mobility, and/or time varying weather conditions). Multiple data streams $X_{i j}(t)$ randomly enter the system, where $X_{i j}(t)$ represents an exogenous process of packets arriving to node $i$ destined for node $j$. Packets are dynamically routed from node to node over multi-hop paths using wireless data links.

Nodes can transmit data over multiple links simultaneously by assigning power to the link for each node pair $(a, b)$ according to a power matrix $\underline{P}(t)=\left(P_{a b}(t)\right)$, subject to a total power constraint at each node. Transmission rates over all link pairs are determined by the power allocation matrix $\underline{P}(t)$ and the current channel state $\underline{S}(t)$ according to a rate-power curve (a)

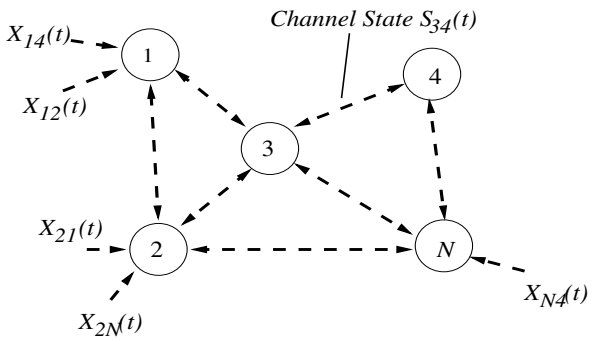

(b)

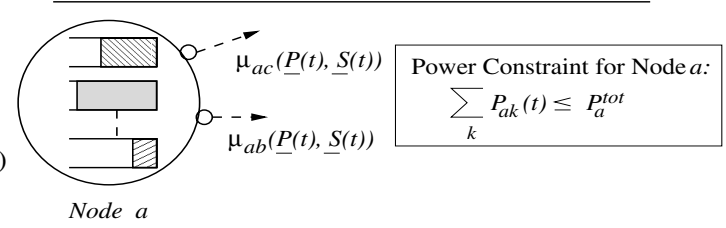

Fig. 1. (a) A wireless network with multiple input streams, and (b) a close-up of one node, illustrating the internal queues.

$\mu(\underline{P}, \underline{S})$. Each node contains $N-1$ internal queues for storing data according to its destination (Fig. 1). A controller allocates power and schedules the data to be routed over the links in reaction to channel state and queue backlog information. The goal of the controller is to stabilize the system and thereby achieve maximum throughput and maintain acceptably low network delay.

We establish the network capacity region: The set of all input rate matrices $\left(\lambda_{i j}\right)$ that the system can stably support (where $\lambda_{i j}$ represents the rate of data entering node $i$ destined for node $j$ ). This region is determined by considering all possible routing and power allocation strategies, and can be expressed in terms of the steady state channel probabilities, the node power constraints, and the rate-power function $\underline{\mu}(\underline{P}, \underline{S})$. We emphasize that this is a network layer notion of capacity, where $\underline{\mu}(\underline{P}, \underline{S})$ is a general function representing the rate achievable on the wireless links under a given physical layer modulation and coding strategy. This is distinct from the information theoretic capacity of the wireless network, which includes optimization over all possible modulation and coding schemes and involves many of the unsolved problems of network information theory. We do not address the information theoretic capacity in this work, and use the term capacity to represent network layer capacity.

We present a joint routing and power allocation policy which stabilizes the system and provides bounded average delay guarantees whenever the input rates are strictly inside the network capacity region. Such performance holds for general Markov modulated arrival and channel state processes, even if the specific channel probabilities and packet arrival rates are unknown to the network controller. The strategy involves solving an optimization problem every timeslot. We implement centralized 
and decentralized approximations of the algorithm for an adhoc wireless network, where channel variations are due to user mobility.

Previous work on power control for wireless systems is found in [1-7], [23], [25-27]. In [1], a stabilizing power allocation strategy is developed for a satellite downlink with random inputs and time varying channels. Routing over finite buffer downlinks is considered in [2]. In [3,4], optimal power allocation policies are developed for minimizing the energy expended to transmit data arriving to a downlink node with a single transmitter. Scheduling and allocation strategies for networks are considered in [5-7], where static power allocation policies are developed to support flows with known traffic rates $\lambda_{i j}$. Game theory approaches to network problems are considered in [810], where decentralized pricing mechanisms are constructed to enable the system to reach a static equilibrium point which maximizes some function of user utility.

Asymptotic analysis of capacity regions for large, static wireless networks is provided in [11,12], and for mobile networks in [13]. Our work is inspired by the approach of Tassiulas in [14], where a Lyapunov drift technique is used to develop a throughput optimal link scheduling policy for a multi-hop packet radio network. Further work on Lyapunov analysis is found in the switching and scheduling literature [16-19].

The main contributions in this paper are the formulation of a general power control problem for time varying wireless networks, the proof of the capacity region, and the development of capacity achieving routing and power allocation algorithms which offer delay guarantees. These algorithms hold for systems with general arrival and channel processes, including adhoc networks with mobility.

In the next section, we introduce the power allocation problem for wireless networks. In Section III we establish the capacity region. In Section IV stabilizing power allocation policies are developed, and in Section V decentralized implementations are developed for networks with independent channels. Finally, we implement both a centralized and a decentralized version of the policy for an ad-hoc wireless network with mobility, and simulate the system to compare with the Grossglauser-Tse relay algorithm of [13].

\section{The System Model}

Consider the $N$ node system of Fig. 1. We represent the channel process by the channel state matrix $\underline{S}(t)=\left(S_{a b}(t)\right)$, where $S_{a b}(t)$ represents the current state of channel $(a, b)$ (representing, for example, attenuation values and/or noise levels). Channels hold their state for timeslots of length $T$, with transitions occurring on slot boundaries $t=k T$. It is assumed that channel states are known at the beginning of each timeslot. Such information can be obtained either through direct measurement (where timeslots are assumed to be long in comparison to the required measurement time) or through a combination of measurement and channel prediction. ${ }^{1}$ The channel process $\underline{S}(t)$ takes values on a finite state space, and is ergodic with time average probabilities $\pi_{\underline{S}}$ for each state $\underline{S}$.

Every timeslot, a controller determines transmission rates by allocating a power matrix $\underline{P}(t)=\left(P_{a b}(t)\right)$ subject to a total

\footnotetext{
${ }^{1}$ Accurate prediction schemes are developed in [20].
}

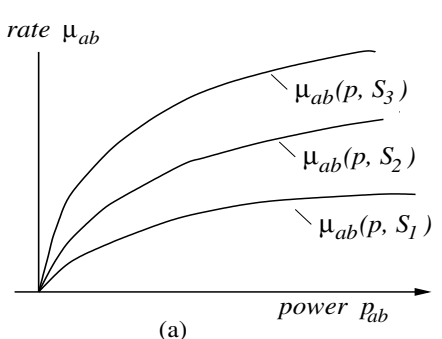

(a) (b)

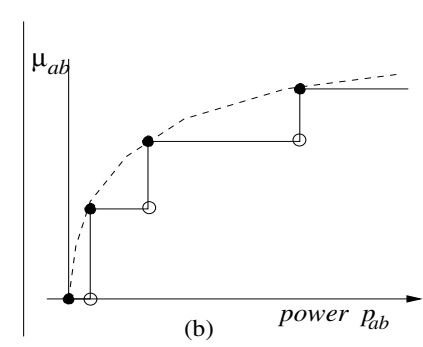

Fig. 2. (a) A set of rate-power curves for improving channel conditions $S_{1}, S_{2}, S_{3}$, and (b) a curve restricted to a finite set of operating points corresponding to full packet transmissions. Curves illustrate behavior on link $(a, b)$ when the single power parameter $P_{a b}$ is increased, in which case the concave increasing profiles are typical.

power constraint $\sum_{b \neq i} P_{i b}(t) \leq P_{i}^{t o t}$ for all nodes $i$. Additional power constraints can be introduced, such as constraints on the number of outgoing links that can be allocated power when a node is transmitting or receiving. It is therefore useful to represent the power constraint in the form $\underline{P}(t) \in \Pi$, where $\Pi$ is a compact set of acceptable power allocations which include the power limits for each node.

Link rates are determined by a corresponding rate-power curve $\underline{\mu}(\underline{P}, \underline{S})=\left(\mu_{a b}(\underline{P}, \underline{S})\right)$ (see Fig. 2). It is assumed that data can be split continuously, so that each timeslot the transmission rate $\mu_{a b}$ determines the number of bits that can be transferred over the wireless link $(a, b)$. Such an assumption is valid if variable length packets can be split and re-packaged with new headers for re-sequencing at the destination (we neglect the extra bits due to such headers in this analysis). Alternately, splitting and relabeling can be avoided altogether if all packets have fixed lengths and the transmission rates $\mu$ are restricted to integral multiples of the packet-length/timeslot quotient $L / T$.

Note that, in general, the transmission rate over a link $(a, b)$ of the network depends on the full matrix of power allocation decisions. This is because communication rates over the link may be influenced by interference from other channels. For example, achievable data rates could be approximated by using the standard CDMA signal-to-interference ratio in the $\log ()$ formula for the capacity of a white Gaussian noise channel:

Example Rate-Power Curve: $\mu_{a b}(\underline{P}, \underline{S})=$

$$
\log \left(1+\frac{\alpha_{a b} P_{a b}}{N_{b}+\frac{\alpha_{a b}}{G_{1}} \sum_{j \neq b} P_{a j}+\frac{1}{G_{2}} \sum_{i \neq a} \alpha_{i b} \sum_{j} P_{i j}}\right)
$$

where $G_{1}, G_{2} \geq 1$ represent the CDMA gain parameters for signals from the same transmitter and different transmitters, respectively, and $N_{b}$ and $\alpha_{i j}$ represent noise and fading coefficients (associated with the particular channel state $\underline{S}$ ).

Alternatively, the $\mu_{a b}()$ curves could represent rate curves for a specific set of coding schemes designed to achieve a sufficiently low probability of error. Note that practical systems rely on a finite databank of codes, and hence may be restricted to a finite set of feasible operating points. In this case, rate-power curves are piecewise constant (see Fig. 2b). In general, we assume only that $\mu(\underline{P}, \underline{S})$ is a piecewise continuous function of power for each channel state $\underline{S}$. More precisely, we assume the function is upper semi-continuous ${ }^{2}$, and hence limits are achieved from above (see [22]).

\footnotetext{
${ }^{2}$ I.e., that $\lim _{\underline{P} \rightarrow \underline{P}^{*}} \mu_{a b}(\underline{P}, \underline{S}) \leq \mu_{a b}\left(\underline{P}^{*}, \underline{S}\right)$ for all $(a, b)$ and all $\underline{S}$.
} 
The general rate-power curve description of a wireless link contains as a special case a wired link with fixed data rate, as the $\mu_{a b}(\underline{P}, \underline{S})$ function can take a constant value for all power levels. Note also that a broken or non-existent link can be modeled by a rate-power curve that is zero for all power levels at one or more channel states. Thus, the general power curve formulation provides the ability to address hybrid networks containing both wireline and wireless components.

\section{Stability And the Network Capacity Region}

Here we develop the region of all data rates for which some power allocation and routing strategy exists to stabilize the network. We consider all possible control strategies, and begin by precisely defining the notion of stability.

\section{A. Stability of Queueing Systems}

Consider a single queue with an input process $X(t)$ and a time varying server process $\mu(t)$. Because the input stream and server process could arise from an arbitrary, potentially nonergodic routing and power allocation policy, our definition of queue stability must be robust to handle all possible arrival and server processes. Let the unfinished work function $U(t)$ represent the amount of unprocessed bits remaining in the queue at time $t$. As a measure of the fraction of time the unfinished work in the queue is above a certain value $M$, we define the following "overflow" function $g(M)$ :

$$
g(M)=\limsup _{t \rightarrow \infty} \frac{1}{t} \int_{0}^{t} 1_{[U(\tau)>M]} d \tau
$$

where the indicator function $1_{E}$ used above takes the value 1 whenever event $E$ is satisfied, and 0 otherwise.

The above limit ${ }^{3}$ always exists, so that $g(M) \in[0,1]$.

Definition 1. A single server queueing system is stable if $g(M) \rightarrow 0$ as $M \rightarrow \infty$.

Notice that if sample paths of unfinished work in the queue are ergodic and a steady state exists, the overflow function $g(M)$ is simply the steady state probability that the unfinished work in the queue exceeds the value $M$. Stability in this case is identical to the usual notion of stability defined in terms of a vanishing complementary occupancy distribution (see $[21,20,14,16,17])$. A network of queues is said to be stable if all individual queues are stable. Consider a network of $K$ queues with unfinished work levels $U_{k}(t), k=1, \ldots, K$, and define:

$$
\begin{aligned}
g_{k}(M) & =\limsup _{t \rightarrow \infty} \frac{1}{t} \int_{0}^{t} 1_{\left[U_{k}(\tau)>M\right]} d \tau \\
g_{\text {sum }}(M) & =\limsup _{t \rightarrow \infty} \frac{1}{t} \int_{0}^{t} 1_{\left[U_{1}(\tau)+\ldots+U_{K}(\tau)>M\right]} d \tau
\end{aligned}
$$

Lemma 1. (Network Stability) For a network of $K$ queues, we have $g_{\text {sum }}(M) \rightarrow 0$ if and only if $g_{k}(M) \rightarrow 0$ for all queues $k \in\{1, \ldots, K\}$. In particular, if the network is stable, then there exists a finite value $M$ such that the unfinished work in all queues simultaneously falls below the value $M$ infinitely often.

\footnotetext{
${ }^{3}$ Where the lim sup of a function $f(t)$ is defined:
} $\lim \sup _{t \rightarrow \infty} f(t)=\lim _{t \rightarrow \infty}\left[\sup _{\tau \geq t} f(\tau)\right]$.
Proof: Note that $1_{\left[\sum_{k} U_{k}(t)>M\right]} \leq \sum_{k} 1_{\left[U_{k}(t)>M / K\right]}$. The lemma then follows easily from the definition of stability and the fact that the lim sup of a sum is less than or equal to the sum of the lim sups.

\section{B. The Capacity Region $\Lambda$}

Here we develop the capacity region of all data rates stabilizable by a wireless network characterized by the following properties:

- An ergodic channel state process $\underline{S}(t)$ with state probabilities $\pi_{\underline{S}}$

- A piecewise continuous rate-power function $\mu(\underline{P}, \underline{S})$

- A power constraint $\underline{P} \in \Pi$ for all $t$ (where $\Pi$ is a compact set of acceptable power allocations)

For convenience, we classify all data flowing through the network as belonging to a particular commodity $c \in\{1, \ldots, N\}$, representing the destination node for the data. Let $X_{i}^{(c)}(t)$ represent the total amount of commodity $c$ bits that arrived to the network at node $i$. We assume the $X_{i}^{(c)}(t)$ process is rate ergodic, so that the following rates are well defined with probability 1:

$$
\lambda_{i c}=\lim _{t \rightarrow \infty} \frac{X_{i}^{(c)}(t)}{t}, \quad(i, c) \in\{1, \ldots, N\}^{2}
$$

Definition 2. The capacity region $\Lambda$ is the closed region of $N \times$ $N$ rate matrices $\left(\lambda_{i j}\right)$ with the following properties:

- $\left(\lambda_{i c}\right) \in \Lambda$ is a necessary condition for network stability, where all possible ergodic or non-ergodic stabilizing power control and routing algorithms are considered (including algorithms which have full knowledge of future events).

- $\left(\lambda_{i c}\right)$ strictly interior to $\Lambda$ is a sufficient condition for the network to be stabilized by a policy which does not have a-priori knowledge of future events.

It is remarkable that such a set exists, and that full knowledge of future events does not expand the region of stabilizable rates. Below we describe the set of rate matrices $\Lambda$ making up this region, and in Theorem 1 we show this set $\Lambda$ is the true capacity region by establishing both the necessary and sufficient conditions listed above.

To understand the capacity region of a wireless network, we first define the network graph family $\Gamma$ :

$$
\Gamma=\sum_{\underline{S}} \pi_{\underline{S}} \text { Convex_Hull }\{\underline{\mu}(\underline{P}, \underline{S}) \mid \underline{P} \in \Pi\}
$$

where addition and scalar multiplication of sets is used. ${ }^{4}$

Thus, a rate matrix $\underline{R}=\left(R_{a b}\right)$ is in graph family $\Gamma$ if and only if $\underline{R}$ can be represented as $\underline{R}=\sum_{\underline{S}} \pi_{\underline{S}} \underline{R}^{\underline{S}}$, where each matrix $\underline{R}^{S}$ is inside the convex hull of the set of rates achievable by power allocation under channel state $\underline{S}$. In the proof of Theorem 1, we show that graph family $\Gamma$ can be viewed as the set of all long-term transmission rates $\left(R_{a b}\right)$ that the network can support on the single-hop wireless links connecting node

\footnotetext{
${ }^{4}$ For sets $A, B$ and scalars $\alpha, \beta$, the set $\alpha A+\beta B$ is defined as $\{\gamma \mid \gamma=\alpha a+\beta b$ for some $a \in A, b \in B\}$.
} 
pairs $(a, b)$. A particular power allocation policy gives rise to a particular rate matrix $\underline{R}=\left(R_{a b}\right)$. Given this matrix, the network can be described as a weighted graph, where weights $R_{a b}$ can be viewed as link capacities in a traditional wireline network.

Network Capacity Region: The capacity region $\Lambda$ is the set of all input rate matrices $\left(\lambda_{i c}\right)$ such that there exist multicommodity flow variables $\left\{f_{a b}^{(c)}\right\}$ satisfying:

$$
\begin{array}{cl}
f_{a b}^{(c)} \geq 0 & \forall a, b, c \\
\lambda_{i c}=\sum_{b} f_{i b}^{(c)}-\sum_{a} f_{a i}^{(c)} & \forall i, c \text { such that } i \neq c \\
\sum_{a} \lambda_{a c}=\sum_{a} f_{a c}^{(c)} & \forall c \\
\left(\sum_{c} f_{a b}^{(c)}\right) \leq\left(R_{a b}\right) & \text { for some }\left(R_{a b}\right) \in \Gamma
\end{array}
$$

where the matrix inequality in (7) is considered entrywise.

Thus, a rate matrix $\left(\lambda_{i c}\right)$ is in the capacity region $\Lambda$ if there exists a point $\left(R_{a b}\right) \in \Gamma$ that defines link capacities in a traditional graph network, such that there exist multi-commodity flow variables $\left\{f_{a b}^{(c)}\right\}$ which support the $\lambda_{i c}$ rates with respect to this graph. Note that (4)-(6) indicate the multi-commodity flow variables $\left\{f_{a b}^{(c)}\right\}$ represent a feasible routing for commodity $c$. Equations (5) and (6) imply that the net influx of commodity $c$ bits is zero at intermediate nodes $i \neq c$, and is equal to $\sum_{a} \lambda_{a c}$ at the destination node $c$.

It can be shown using standard convex analysis techniques [22] that the set $\Gamma$ is convex, and that $\Lambda$ is compact and convex. Such structural properties are used in the proof of the following theorem.

Theorem 1. (Capacity Region for a Wireless Network) (a) A necessary condition for stability is $\left(\lambda_{i c}\right) \in \Lambda$. (b) If arrivals and channel state variations are Markov modulated on a finite state space, a sufficient condition for stability is that $\left(\lambda_{i c}\right)$ is strictly interior to $\Lambda$.

Proof: A full proof of (a) is given in Appendix A, where it is shown that no control algorithm can achieve stability beyond the set $\Lambda$, even if the entire set of future events is known in advance. Part (b) can be shown constructively by routing data according to the flow variables $\left\{f_{a b}^{(c)}\right\}$ and allocating power to meet the long-term link capacity requirements $\left(R_{a b}\right)$ (where the $f_{a b}^{(c)}$ and $R_{a b}$ values correspond to the input rate matrix $\left(\lambda_{i j}\right)$ via (4)-(7)). For brevity, we omit a full proof here (the reader is referred to [23]). In the next section, stability analysis of such a policy is shown when the arriving data has bounded second moments, and a bound on average delay is provided when arrivals and channel states are iid over timeslots.

\section{A Stabilizing Policy}

In the previous section we described the capacity region $\Lambda$ in terms of flow variables $f_{a b}^{(c)}$ and a link matrix $\left(R_{a b}\right) \in \Gamma$ which satisfy (4)-(7). In principle, these values can be computed if the arrival rates $\left(\lambda_{i c}\right)$ and channel probabilities $\pi_{\underline{S}}$ are known in advance. This allows us to view power allocation and routing in a decoupled manner, where data is routed according to flow variables $f_{a b}^{(c)}$, and power is allocated to achieve long-term link capacities $\left(R_{a b}\right)$. Here we construct such a policy and show it provides a bound on average delay. We then use this analysis to construct a more practical and robust strategy which offers similar performance without requiring knowledge of the input and channel statistics. We start by presenting a preliminary lemma which makes use of a well developed theory of Lyapunov drift (see $[21,17,18,16,14])$.

\section{A. Lyapunov Drift}

Let $\underline{U}(t)=\left(U_{i}^{(c)}(t)\right)$ represent the matrix of unfinished work in the wireless network, where $\left(U_{i}^{(c)}(t)\right)$ represents the amount of commodity $c$ bits in the output queue of node $i$. Define a non-negative function $L(\underline{U})$ of the unfinished work matrix $\underline{U}$. Below we present a simple condition which guarantees network stability and provides a performance bound. The lemma combines the steady state analysis for Lyapunov drift presented in [21] and the delay analysis in [17] into a simple statement useful for stability and performance analysis in our wireless network.

Lemma 2. (Lyapunov Drift) If the Lyapunov function of unfinished work $L(\underline{U})$ satisfies:

$$
\mathbb{E}\{L(\underline{U}(t+T))-L(\underline{U}(t)) \mid \underline{U}(t)\} \leq B-\sum_{i, c} \theta_{i}^{(c)} U_{i}^{(c)}(t)
$$

for positive constants $B,\left\{\theta_{i}^{(c)}\right\}$, then:

$$
\limsup _{M \rightarrow \infty} \sum_{i, c} \theta_{i}^{(c)}\left[\frac{1}{M} \sum_{k=0}^{M-1} \mathbb{E}\left\{U_{i}^{(c)}(k T)\right\}\right] \leq B
$$

Furthermore, if there is a nonzero probability that the system will eventually empty, then a steady state distribution for unfinished work exists, with bounded average occupancies $\bar{U}_{i}^{(c)}$ satisfying $\sum_{i, c} \theta_{i}^{(c)} \bar{U}_{i}^{(c)} \leq B$.

Proof: Taking expectations of (8) over the distribution of $\underline{U}(k T)$ and summing over $k$ from 0 to $M-1$ yields:

$$
\begin{aligned}
& \mathbb{E}\{L(\underline{U}(M T))\}- \mathbb{E}\{L(\underline{U}(0))\} \leq \\
& B M-\sum_{k=0}^{M-1} \sum_{i, c} \theta_{i}^{(c)} \mathbb{E}\left\{U_{i}^{(c)}(k T)\right\}
\end{aligned}
$$

Hence, by a simple telescoping series argument similar to the technique used in [17], we have:

$$
\frac{1}{M} \sum_{k=0}^{M-1}\left[\sum_{i, c} \theta_{i}^{(c)} \mathbb{E}\left\{U_{i}^{(c)}(k T)\right\}\right] \leq B+\mathbb{E}\{L(\underline{U}(0))\} / M
$$

Taking the lim sup of the above inequality as $M \rightarrow \infty$ yields (9). If there is a non-zero probability the system empties ${ }^{5}$, standard Lyapunov drift techniques $[21,18,17,14]$ and renewal theory [24] can be used together with (9) to establish the existence of a steady state unfinished work matrix satisfying the given inequality.

\footnotetext{
${ }^{5}$ The requirement of a non-zero probability that the system empties is necessary to deal with the uncountably infinite state space of unfinished work, similar to the treatment in [1].
} 


\section{B. Stability for Known Arrival and Channel Statistics}

We now construct a simple policy for stabilizing the system based on the $f_{a b}^{(c)}$ and $R_{a b}$ values associated with a known rate matrix $\left(\lambda_{i j}\right)$ and known channel probabilities $\pi_{\underline{S}}$. The policy is not offered as a practical means of network control, but as a baseline by which other algorithms can be compared. We first demonstrate that power can be allocated so that the long-term link capacities of the network converge to the matrix $\left(R_{a b}\right)$.

Lemma 3. (Graph Family Achievability) Let $\left(R_{a b}\right)$ be a matrix within the graph family $\Gamma$ (defined in (3)). A power control algorithm which yields

$$
\left(\lim _{t \rightarrow \infty} \frac{1}{t} \int_{0}^{t} \mu_{a b}(\underline{P}(\tau), \underline{S}(\tau)) d \tau\right)=\left(R_{a b}\right)
$$

can be achieved by a stationary randomized policy, where every timeslot the channel state $\underline{S}(t)$ is observed and a power matrix is chosen randomly from a finite set of $k$ allocations $\left\{\underline{P}_{1}^{S}, \ldots, \underline{P} \underline{\underline{S}}\right\}$ according to probabilities $\left(q_{1}^{\frac{S}{1}}, \ldots, q \frac{S}{k}\right)$.

Proof: Because $\left(R_{a b}\right) \in \Gamma$, by (3) there exist matrices $\underline{R}^{\underline{S}}$ such that:

$$
\sum_{\underline{S}} \pi_{\underline{S}} \underline{R}^{\underline{S}}=\left(R_{a b}\right)
$$

where each $\underline{R}^{\underline{S}}$ is in Convex_Hull $\{\mu(\underline{P}, \underline{S}) \mid \underline{P} \in \Pi\}$. By Caratheodory's Theorem [22], any point $\underline{R}^{\underline{S}}$ in the convex hull of the set $\{\mu(\underline{P}, \underline{S}) \mid \underline{P} \in \Pi\}$ can be expressed as a finite combination of matrices:

$$
\underline{R}^{\underline{S}}=q \underline{\underline{S}} \underline{R}_{1}^{S}+\ldots+q \frac{S}{k} \underline{R}_{k}^{S}
$$

where the $\left\{q_{i}^{S}\right\}$ values are nonnegative numbers that sum to 1 which represent probabilities for the randomized algorithm, and $\underline{R}_{i}^{S} \in\{\mu(\underline{P}, \underline{S}) \mid \underline{P} \in \Pi\}$ for each $i$. Choosing power allocations $\{\underline{\underline{P}} \overline{\underline{S}}, \ldots, \underline{P} \underline{\underline{S}}\}$ such that $\mu\left(\underline{P}_{i}^{\underline{S}}, \underline{S}\right)=\underline{R}_{i}^{S}$ and allocating power according to the randomized policy ensures $\mathbb{E}\{\underline{\mu}(\underline{P}(t), \underline{S}(t)) \mid \underline{S}(t)=\underline{S}\}=\underline{R}^{S}$ (where the expectation is taken over the $\left\{q_{i}^{S}\right\}$ probabilities). Integrating the resulting $\underline{\mu}(\underline{P}(t), \underline{S}(t))$ function, we have:

$$
\frac{1}{t} \int_{0}^{t} \underline{\mu}(\underline{P}(\tau), \underline{S}(\tau)) d \tau=\sum_{\underline{S}} \frac{\left\|T_{\underline{S}(t)}\right\|}{t} \mathbb{E}_{\text {empirical }}\{\underline{\mu} \mid \underline{S}\}
$$

where $\left\|T_{\underline{S}(t)}\right\|$ represents the total time the system is in channel state $\underline{S}$ during $[0, t]$, and $\mathbb{E}_{\text {empirical }}\{\underline{\mu} \mid \underline{S}\}$ represents the empirical average rate given channel state $\underline{S}$. Taking limits of the above equality and using ergodicity together with (11) yields (10).

It can be shown that if the set $\{\mu(\underline{P}, \underline{S}) \mid \underline{P} \in \Pi\}$ is convex for each channel state $\underline{S}$, then then (10) can be achieved by a non-random policy which chooses a fixed power matrix $\underline{\underline{P}} \underline{\underline{S}}$ whenever in channel state $\underline{S}$ (see [23]). Note that the policy of Lemma 3 bases decisions only on the current channel state, and does not depend on queue backlogs. However, the policy is rather idealized: The existential nature of Lemma 3 does not provide any practical means of computing the power values and probabilities needed to implement the policy. However, this allocation policy is analyzable and useful for comparison with other algorithms. Below we develop a control strategy based on this idealized policy. The values $f_{a b}^{(c)}$ and $R_{a b}$ are assumed to be known.

\section{Randomized Policy for Known System Statistics:}

Power Allocation: Every timeslot, observe the channel state $\underline{S}$ and allocate power according to the algorithm of Lemma 3, achieving the long-term link capacity matrix $\left(R_{a b}\right)$ with instantaneous link rates $\mu_{a b}(t)$.

Routing: For every link $(a, b)$ such that $\sum_{c} f_{a b}^{(c)}>0$, transmit commodity $c_{a b}$, where $c_{a b}$ is chosen randomly with probability $f_{a b}^{(c)} / \sum_{c} f_{a b}^{(c)}$. However, use only a fraction $\frac{\sum_{c} f_{a b}^{(c)}}{R_{a b}}$ of the instantaneous link rate, so that only $T \mu_{a b}(t) \frac{\sum_{c} f_{a b}^{(c)}}{R_{a b}}$ bits are delivered. If a node does not have enough (or any) bits of a certain commodity to send over its output links, null bits are delivered, so that links have idle times which are not used by other commodities.

For simplicity of exposition, we analyze the above strategy assuming arrivals and channel states are independent from timeslot to timeslot (a modified analysis to address Markovian dynamics is presented in Subsection $E$ ). Every timeslot, channels independently transition to state $\underline{S}(t)$ with probability $\pi_{S}$. Additionally, every timeslot new bits from all commodities $c$ independently enter the network as an arrival matrix $\underline{A}(t)=\left(A_{i c}(t)\right)$ with distribution $f(\underline{A})$ and expectation $\mathbb{E}\left\{\left(A_{i c}\right) / T\right\}=\left(\lambda_{i c}\right)$. Suppose the rate matrix $\left(\lambda_{i c}\right)$ is strictly interior to the capacity region $\Lambda$, so that there is a positive value $\epsilon$ that can be added to each component of $\left(\lambda_{i c}\right)$ such that $\left(\lambda_{i c}+\epsilon\right) \in \Lambda$. Let $\left(R_{a b}\right)$ and $\left\{f_{a b}^{(c)}\right\}$ represent the network graph and multi-commodity flow variables, respectively, associated with rates $\left(\lambda_{i c}+\epsilon\right)$ and satisfying (4)-(7). In particular:

$$
\begin{gathered}
\left(\lambda_{i c}+\epsilon\right)=\sum_{b} f_{i b}^{(c)}-\sum_{a} f_{a i}^{(c)} \text { for } i \neq c \\
\left(\sum_{c} f_{a b}^{(c)}\right) \leq\left(R_{a b}\right)
\end{gathered}
$$

We define $\mu_{a b}(t)$ as the total rate offered on link $(a, b)$ for the timeslot beginning at time $t$, and define $\mu_{a b}^{(c)}(t)$ as the transmission rate offered to commodity $c$ traffic, noting that only a fraction of the full rate is used on any transmission (so that $\mu_{a b}^{(c)}(t)=\mu_{a b}(t) \frac{\sum_{c} f_{a b}^{(c)}}{R_{a b}}$ with probability $f_{a b}^{(c)} / \sum_{c} f_{a b}^{(c)}$, and zero otherwise). Using (10) and the fact that channel states are iid, we find that every timeslot the expected rates satisfy:

$$
\begin{aligned}
\mathbb{E}\left\{\mu_{a b}(t)\right\} & =R_{a b} \\
\mathbb{E}\left\{\mu_{a b}^{(c)}(t)\right\} & =f_{a b}^{(c)}
\end{aligned}
$$

where the expectation is taken over the random channel state and the random power allocation of the above control policy. Thus, the control policy is designed to offer expected transmission rates equal to the multi-commodity flow variables $\left\{f_{a b}^{(c)}\right\}$.

Theorem 2. (Stabilizing Policy for Known Statistics) Consider an $N$ node wireless network (with timeslot independence properties of arrivals and channel states as described above), with capacity region $\Lambda$ and input rates $\left(\lambda_{i c}\right)$ such that $\left(\lambda_{i c}+\epsilon\right) \in \Lambda$ 
for some $\epsilon>0$. Then, jointly routing and allocating power according to the above randomized policy stabilizes the system and guarantees bounded average bit occupancies $\bar{U}_{i}^{(c)}$ satisfying:

$$
\sum_{i, c} \bar{U}_{i}^{(c)} \leq \frac{T B N}{\epsilon}
$$

where

$$
B=\frac{1}{2 N} \sum_{i, c} \mathbb{E}\left\{\left(A_{i c} / T\right)^{2}\right\}+\frac{\left(\mu_{\max }^{\text {out }}+\mu_{\max }^{\text {in }}\right)^{2}}{2}
$$

where $\mu_{\max }^{\text {in }}$ and $\mu_{\text {max }}^{\text {out }}$ represent the maximum bit rate into and out of a node, respectively, over all channel states.

Proof: Let $A_{i c}(t)$ represent the new commodity $c$ bits arriving to source $i$ at the beginning of a timeslot $t$, and let $\mu_{a b}^{(c)}(t)$ represent the rate offered to commodity $c$ over the $(a, b)$ link under the given power allocation algorithm. In terms of these variables, the one-step dynamics of unfinished work satisfies for all $i \neq c$ :

$$
\begin{array}{r}
U_{i}^{(c)}(t+T) \leq \max \left(U_{i}^{(c)}(t)-T \sum_{b} \mu_{i b}^{(c)}(t), 0\right)+ \\
T \sum_{a} \mu_{a i}^{(c)}(t)+A_{i c}(t)
\end{array}
$$

where (18) holds as an inequality instead of an equality because the total bits arriving to node $i$ from other nodes of the network may be less than $T \sum_{a} \mu_{a i}^{(c)}(t)$ if these other nodes have little or no data to send.

Now define the Lyapunov function $L(\underline{U})=\sum_{i \neq c}\left[U_{i}^{(c)}\right]^{2}$. For ease of notation, we neglect the time subscripts and represent $\mu_{a b}^{(c)}(t)$ and $A_{i c}(t)$ as $\mu_{a b}^{(c)}$ and $A_{i c}$. Squaring both sides of (18) and noting that $\max ^{2}(x, 0) \leq x^{2}$, we have:

$$
\begin{aligned}
& {\left[U_{i}^{(c)}(t+T)\right]^{2}-\left[U_{i}^{(c)}(t)\right]^{2} \leq T^{2}\left[A_{i c} / T\right]^{2}+} \\
& \quad T^{2}\left[\left(\sum_{b} \mu_{i b}^{(c)}\right)^{2}+\left(\sum_{a} \mu_{a i}^{(c)}\right)^{2}+2 \frac{A_{i c}}{T}\left(\sum_{a} \mu_{a i}^{(c)}\right)\right]+ \\
& \quad-2 T U_{i}^{(c)}(t)\left[\sum_{b} \mu_{i b}^{(c)}-\sum_{a} \mu_{a i}^{(c)}-\left(A_{i c} / T\right)\right]
\end{aligned}
$$

Summing (19) over all nodes $i$ and commodities $c \neq i$ and taking expectations (noting that $\mathbb{E}\left\{A_{i c} / T\right\}=\lambda_{i c}$ ), it follows that:

$$
\begin{aligned}
& \mathbb{E}\{L(\underline{U}(t+T))-L(\underline{U}(t)) \mid \underline{U}(t)\} \leq 2 T^{2} B N+ \\
& -2 T \sum_{i \neq c} U_{i}^{(c)}(t)\left[\mathbb{E}\left\{\sum_{b} \mu_{i b}^{(c)}-\sum_{a} \mu_{a i}^{(c)}\right\}-\lambda_{i c}\right]
\end{aligned}
$$

where $B$ is defined in (17). Note that the $B$ constant used above was obtained from the second term on the right hand side of (19) by using the Cauchy-Schwartz inequality for sums and observing that $\sum_{c} \lambda_{i c} \leq \mu_{\text {max }}^{\text {out }}$. The remaining expectation in (20) is taken over all possible random channel states, and from (15) we know $\mathbb{E}\left\{\mu_{a b}^{(c)}\right\}=f_{a b}^{(c)}$. Hence, the flow variables can be directly inserted into (20). These multi-commodity flows were designed to satisfy rates $\left(\lambda_{i c}+\epsilon\right)$, hence, directly applying the multi-commodity flow condition (12) in the expectation of (20) yields:

$$
\begin{aligned}
\mathbb{E}\{L(\underline{U}(t+T))-L(\underline{U}(t)) \mid \underline{U}(t)\} \leq \\
2 T^{2} B N-2 T \epsilon \sum_{i \neq c} U_{i}^{(c)}(t)
\end{aligned}
$$

Applying the Lyapunov Drift Lemma (Lemma 2) to the above inequality and noting that $U_{i}^{(i)}=0$ for all $i$ proves the result.

\section{A Dynamic Policy for Unknown System Statistics}

The stabilizing policy of the above section requires full knowledge of arrival rates and channel state probabilities, along with the associated multi-commodity flows and the randomized power allocations. Here we present a dynamic power control and routing scheme which requires no knowledge of the arrival rates or channel model, yet performs better than the previous policy which does use this information. This surprising result arises because the dynamic policy considers both the channel state $\underline{S}(t)$ and the system backlogs $\underline{U}(t)$ when making control decisions. The policy is inspired by the maximum weighted matching algorithms developed by Tassiulas in [14] for stable server scheduling in a multi-hop radio network and an $N \times N$ packet switch, and generalizes the Tassiulas algorithm by considering power allocation with general interference and time varying channel characteristics. Every timeslot the network controller observes the channel state $\underline{S}(t)$ and the matrix of queue backlogs $\underline{U}(t)=\left(U_{i}^{(c)}(t)\right)$ and performs routing and power control as follows.

Dynamic Routing and Power Control (DRPC) Policy:

1) For all links $(a, b)$, find commodity $c_{a b}^{*}$ such that:

$$
c_{a b}^{*}=\underset{c \in\{1, \ldots, N\}}{\arg \max }\left\{U_{a}^{(c)}(t)-U_{b}^{(c)}(t)\right\}
$$

and define: $W_{a b}^{*}=\max \left[U_{a}^{\left(c_{a b}^{*}\right)}(t)-U_{b}^{\left(c_{a b}^{*}\right)}(t), 0\right]$

2) Power Allocation: Choose a matrix $\underline{P}(t)$ such that:

$$
\underline{P}(t)=\underset{\underline{P} \in \Pi}{\arg \max } \sum_{a, b} \mu_{a b}(\underline{P}, \underline{S}(t)) W_{a b}^{*}
$$

3) Routing: Over link $(a, b)$, send an amount of bits from commodity $c_{a b}^{*}$ according to the rate offered by the power allocation. If any node does not have enough bits of a particular commodity to send over all its outgoing links requesting that commodity, null bits are delivered.

Note that the $W_{a b}^{*}$ values represent the maximum differential backlog of commodity $c$ bits between nodes $a$ and $b$. The policy thus uses backpressure to find an optimal routing. Retaining the independence assumptions on arrivals and channels from slot to slot, we have:

Theorem 3. (Stabilizing Policy for Unknown System Statistics) Suppose an $N$-node wireless network has capacity region $\Lambda$ and rate matrix $\left(\lambda_{i c}\right)$ such that $\left(\lambda_{i c}+\epsilon\right) \in \Lambda$ for some $\epsilon>0$, although these rates and the channel probabilities $\pi_{\underline{S}}$ are unknown to the network controller. Then, jointly routing and allocating power according to the above DRPC policy stabilizes the 
system and guarantees bounded average bit occupancies $\bar{U}_{i}^{(c)}$ satisfying:

$$
\sum_{i, c} \bar{U}_{i}^{(c)} \leq \frac{T B N}{\epsilon}
$$

where $B$ is defined in (17).

Note that the performance bound of Theorem 3 is identical to the bound of Theorem 2. However, the bound is established by showing that the dynamic policy performs better than the previous policy.

Proof: Again define the Lyapunov function $L(\underline{U})=$ $\sum_{i, c}\left[U_{i}^{(c)}\right]^{2}$. The proof of Theorem 2 can be followed up to (20) to show the Lyapunov drift satisfies:

$$
\begin{array}{r}
\mathbb{E}\{L(\underline{U}(t+T))-L(\underline{U}(t)) \mid \underline{U}(t)\} / 2 \leq T^{2} B N+ \\
-T \sum_{i \neq c} U_{i}^{(c)}(t)\left[\mathbb{E}\left\{\sum_{b} \mu_{i b}^{(c)}-\sum_{a} \mu_{a i}^{(c)} \mid \underline{U}(t)\right\}-\lambda_{i c}\right]
\end{array}
$$

We compare the above drift for the DRPC policy with the corresponding drift of the randomized policy of Theorem 2, and show that the DRPC policy produces a more negative drift for each $\underline{U}(t)$. To show this, first note that $U_{i}^{(i)}=0$ for all time, and hence the $i \neq c$ condition in the sum of (24) can be removed. We then switch the sums in (24) to express the portion of the drift term that depends on the power allocations $\mu_{a b}^{(c)}(t)$ as follows:

$$
\begin{array}{r}
\sum_{i, c} U_{i}^{(c)}(t) \mathbb{E}\left\{\sum_{b} \mu_{i b}^{(c)}(t)-\sum_{a} \mu_{a i}^{(c)}(t) \mid \underline{U}(t)\right\}= \\
\sum_{a b} \sum_{c} \mathbb{E}\left\{\mu_{a b}^{(c)} \mid \underline{U}(t)\right\}\left[U_{a}^{(c)}(t)-U_{b}^{(c)}(t)\right]
\end{array}
$$

The drift of the DRPC policy and the randomized policy of Theorem 2 can be expressed by using their respective $\mathbb{E}\left\{\mu_{a b}^{(c)}(t) \mid \underline{U}(t)\right\}$ values in (25) and (24). Define $\underline{P}_{S, U}^{*}$ as the power matrix assigned by the DRPC algorithm of (2) given backlog and channel state matrices $\underline{U}$, and $\underline{S}$. For comparison, we have:

$\underline{D R P C}:$

$$
\mathbb{E}\left\{\mu_{a b}^{(c)}(t) \mid \underline{U}(t)\right\}= \begin{cases}\sum_{\underline{S}} \pi_{\underline{S}} \mu_{a b}\left(\underline{P_{\underline{S}}, \underline{U}}, \underline{S}\right) & \text { if } c=c_{a b}^{*} \\ 0 & \text { if } c \neq c_{a b}^{*}\end{cases}
$$

\section{Randomized Algorithm for Known Statistics:}

$$
\mathbb{E}\left\{\mu_{a b}^{(c)}(t) \mid \underline{U}(t)\right\}=f_{a b}^{(c)}
$$

where the $f_{a b}^{(c)}$ values correspond to an associated $\left(R_{a b}\right)$ matrix (see (13),(15)). Comparing the drift terms, we have:

$$
-\sum_{a b} \sum_{c} f_{a b}^{(c)}\left[U_{a}^{(c)}-U_{b}^{(c)}\right] \geq-\sum_{a b}\left(\sum_{c} f_{a b}^{(c)}\right) W_{a b}^{*}
$$

$$
\begin{aligned}
& \geq-\sum_{a b} R_{a b} W_{a b}^{*} \\
&=-\sum_{a b}\left(\sum_{\underline{S}} \pi_{\underline{S}} R_{a b}^{\underline{S}}\right) W_{a b}^{*} \\
& \geq-\sum_{\underline{S}} \pi_{\underline{S}} \max _{\left(R_{a b}^{\underline{S}}\right) \in}\left\{\sum_{a b} R_{a b}^{\underline{S}} W_{a b}^{*}\right\} \\
&\left.\left.\geq-\sum_{\underline{S}} \pi_{\underline{S}} \operatorname{man}_{\underline{P} \in \underline{\Pi}} \sum_{a b} \mu_{a b}, \underline{\underline{S}}\right) \mid \underline{P} \in \Pi\right\} \\
&=-\sum_{a b} \sum_{\underline{S}} \pi_{\underline{S}} \mu_{a b}\left(\underline{P}_{\underline{S}, \underline{U}}^{*}, \underline{S}\right) W_{a b}^{*} \\
&=-\sum_{a b} \sum_{c} \mathbb{E}_{D R P C}\left\{\mu_{a b}^{(c)}(t) \mid \underline{U}(t)\right\} W_{a b}^{*} \\
&=-\sum_{a b} \sum_{c} \mathbb{E}_{D R P C}\left\{\mu_{a b}^{(c)}(t) \mid \underline{U}(t)\right\}\left[U_{a}^{(c)}-U_{b}^{(c)}\right]
\end{aligned}
$$

where (27) follows from (13), and (30) follows from (29) by noting that maximizing a linear function over the convex hull of a compact set ${ }^{6}$ is achieved at a point within the set itself [22]. Equation (33) follows because the DRPC policy clearly chooses $\mu_{a b}^{c}(t)=0$ for all commodities $c$ if $\left[U_{a}^{(c)}-U_{b}^{(c)}\right] \leq 0$. The term in (33) is an expression for the drift for the DRPC policy. Hence, the Lyapunov drift under the DRPC policy is more negative than the drift from the randomized policy of Theorem 2. Thus, the same drift bound in (21) applies, which proves the theorem.

The asymptotic behavior of the performance bound (23) is worth noting. The bound grows asymptotically like $1 / \epsilon$ as the data rates are increased, where $\epsilon$ can be viewed as the 'distance' measure of the rate matrix to the boundary of the capacity region. Such behavior is characteristic of queueing systems, as exemplified by the standard equation for average delay in an M/G/1 queue [24].

Note that $\epsilon$ is a quantity added to each of the $N^{2}$ terms of the rate matrix so that $\left(\lambda_{i c}+\epsilon\right) \in \Lambda$, and hence $\epsilon$ must decrease as the number of users $N$ in the network scales. To measure performance as a function of $N$, it is appropriate to hold the parameter $\delta=N \epsilon$ constant, where $\delta$ can be viewed as the Euclidean distance to the boundary of the capacity region (see [19]). In this way, we can use the performance bound (23) together with Little's Theorem to obtain a bound on average bit delay: $\bar{D}_{b i t} \leq T B N /\left(\lambda_{a v} \delta\right)$, where $\lambda_{a v}=\frac{1}{N} \sum_{i j} \lambda_{i j}$ is the average rate transmitted by a user $i$. In a static network such as that given by the Gupta-Kumar model ([11], [12]), the data rate $\lambda_{a v}$ necessarily decreases as $O(1 / \sqrt{N})$, and hence for a fixed distance $\delta$ from the boundary of the capacity region, the above bound guarantees an average bit delay of $O\left(N^{3 / 2}\right) / \delta$.

\section{Enhanced DRPC}

The DRPC algorithm stabilizes the network by making use of back-pressure, where packets find their way to destinations by

\footnotetext{
${ }^{6}$ Although the set $\operatorname{Conv}\{\underline{\mu}(\underline{P}, \underline{S}) \mid \underline{P} \in \Pi\}$ is not necessarily compact if the $\mu()$ function is not continuous, upper-semicontinuity implies compactness of the set of all rate matrices which are entrywise less than or equal to a matrix
} within this set, and this is sufficient to establish (30). 
moving in directions of decreasing backlog. However, when the network is lightly loaded, packets may take many false turns, which could lead to significant delay for large networks. Performance can often be improved by using the DRPC algorithm with a restricted set of desirable routes for each commodity. However, restricting the routes in this way may reduce network capacity, and may be harmful in time varying situations where networks change and links fail.

Alternatively, we can keep the full set of routes, but program a bias into the DRPC algorithm so that, in low loading situations, nodes are inclined to route packets in the direction of their destinations. We use this idea in the following Enhanced DRPC algorithm, defined in terms of constants $\theta_{i}^{c}>0$ and $V_{i}^{c} \geq 0$.

Enhanced DRPC Algorithm: For all links $(a, b)$, find commodity $c_{a b}^{*}$ such that:

$$
c_{a b}^{*}=\underset{c \in\{1, \ldots, N\}}{\arg \max }\left\{\theta_{a}^{c}\left(U_{a}^{(c)}(t)+V_{a}^{c}\right)-\theta_{b}^{c}\left(U_{b}^{(c)}(t)+V_{b}^{c}\right)\right\}
$$

and define:

$$
W_{a b}^{*}=\theta_{a}^{c_{a b}^{*}}\left(U_{a}^{c_{a b}^{*}}(t)+V_{a}^{c_{a b}^{*}}\right)-\theta_{b}^{c_{a b}^{*}}\left(U_{b}^{c_{a b}^{*}}(t)+V_{b}^{c_{a b}^{*}}\right)
$$

Power allocation and routing is then done as before, solving the optimization problem (22) with respect to these new $W_{a b}^{*}$ values.

The Enhanced DRPC algorithm can be shown to be stabilizing and to offer a delay bound for any constants $\theta_{i}^{c}>0$ and $V_{i}^{c} \geq 0$, while supporting the following services.

Shortest Path Service: Define biases $V_{i}^{c}$ to be the distance (or number of hops) between node $i$ and node $c$ along the shortest path through the network (where $V_{i i}=0$ for all $i$ ). These distances can either be estimated or computed by running a shortest path algorithm. (It is useful to scale these distances by the maximum transmission rate of any node to one of its neighbors.) With these bias values, packets are inclined to move in the direction of their shortest paths - providing low delay in lightly loaded conditions while still ensuring stability throughout the entire capacity region.

Priority Service: The weights $\theta_{i}^{c}$ of the DRPC algorithm can be used to offer priority service to different customers, where a large $\theta_{i}^{c}$ value gives high priority to commodity $c$ packets in node $i$. We note that these values need not be constant, but can be varied in time. Using Lemma 2, it can be shown that dynamically varying the weights such that $\theta_{i}^{c}(t) \in\left[\theta_{\min }, \theta_{\max }\right]$ still ensures network stability, with a performance guarantee of:

$$
\limsup _{K \rightarrow \infty} \frac{1}{K} \sum_{k=0}^{K} \mathbb{E}\left\{\sum_{i, c} \theta_{i}^{c}(k T) U_{i}^{(c)}(k T)\right\} \leq \frac{T B N \theta_{\max }}{\epsilon}
$$

where, formally, the expectation above is taken assuming $\theta_{i}^{c}(k T)$ values are known in advance.

\section{E. Markovian Inputs}

The DRPC policy can be shown to stabilize the system under general Markov modulated channel and arrival processes. Specifically, suppose these processes are modulated by a finite state Markov chain $M(t) \in\{1, \ldots, Y\}$. When the chain is in state $m$ at the start of a timeslot, arrivals $\underline{A}(t)$ enter the system with distribution $f_{m}(\underline{A})$, and channel states $\underline{S}(t)$ are chosen according to probability mass function $f_{m}(\underline{S})$. We assume the Markov chain is ergodic so that time average arrival rates and channel state probabilities converge to $\left(\lambda_{i j}\right)$ and $\pi_{\underline{S}}$, respectively. Hence, for any small value $\delta>0$, we can find an integer $K$ such that time averages of the channel and arrival processes over $K$ timeslots are within $\delta$ of their steady state values, regardless of the initial state of the Markov Chain. Lyapunov analysis similar to Theorems 2 and 3 for the iid case can be used in this Markov modulated context by considering a group of $K$ timeslots as a 'super-timeslot.' Similar analysis has been used in [15] for link scheduling in single-hop networks with Markovian channel conditions.

Notice that the performance bound for iid inputs in (23) is linear in the timeslot length. Correspondingly, bounds for Markovian inputs are linear in the super-timeslot length $K T$. The proof of this fact is omitted for brevity. The interested reader is referred to [23].

\section{Distributed IMPLEMENTATION}

The DRPC algorithm of the previous section involves solving a constrained optimization problem every timeslot, where current channel state and queue backlogs appear as parameters in the optimization. Here we consider decentralized implementations, where users attempt to maximize the weighted sum of data rates in (22) by exchanging information with their neighbors. The current neighbors of a node $i$ are the nodes whose transmissions can be detected at node $i$. We assume that nodes have knowledge of the link conditions between themselves and their neighbors, and are informed of the queue backlogs of their neighbors via a low bandwidth control channel.

\section{A. Networks with Independent Channels}

Consider a network with independent channels, so that the transmission rate on any given link $(a, b)$ depends only on the local link parameters: $\mu_{a b}(\underline{P}, \underline{S})=\mu_{a b}\left(P_{a b}, S_{a b}\right)$. Assume that the rate functions $\mu_{a b}\left(P_{a b}, S_{a b}\right)$ are concave in the single power variable $P_{a b}$ for every channel state $S_{a b}$ (representing diminishing returns in data rate for each incremental increase in power). These assumptions are valid when all links use orthogonal coding schemes, beamforming, and/or when links are spacially separated such that channel interference is negligible.

In this case, the optimization problem (22) has a simple decoupling property, where nodes make independent power control decisions based only on local information. For each node $n \in\{1, \ldots, N\}$, we have the problem of maximizing $\sum_{b} \mu_{n b}\left(P_{n b}, S_{n b}\right)$ subject to the power constraint $\sum_{b} P_{n b} \leq$ $P_{n}^{\text {tot }}$. This optimization is a standard problem of concave maximization subject to a simplex constraint, and can be solved easily in real time with any degree of accuracy. Its solution proceeds according to the standard water-filling arguments, where power is allocated to equalize scaled derivatives of the $\mu_{n b}\left(P_{n b}, S_{n b}\right)$ function for a subset of users with the best channel conditions. 


\section{B. Distributed Approximation for Networks with Interference}

Consider a network with rate-power curves described by the $\log (1+S I R)$ function given in (1). This network has dependent, interfering channels, and the associated optimization problem (22) is nonlinear, non-convex, and difficult to solve even in a centralized manner. Here we provide a simple decentralized approximation, where nodes use a portion of each timeslot to exchange control information with neighbors:

1) At the beginning of a timeslot, each node randomly decides to either transmit at full power $P_{t o t}$ or remain idle, with probability $1 / 2$ for either decision. A control signal of power $P_{t o t}$ is transmitted.

2) Define $\Omega$ as the set of all transmitting nodes. Each node $b$ measures its total resulting interference $\sum_{i \in \Omega} \alpha_{i b} P_{t o t}$, and sends this scalar quantity over a control channel to all neighbors.

3) Using knowledge of the interference, attenuation values, and queue backlogs associated with all neighboring nodes, each transmitting user $a$ decides to transmit using full power to the single neighbor $b$ who maximizes the function:

$$
W_{a b}^{*} \log \left(1+\frac{\alpha_{a b} P_{t o t}}{N_{b}+\frac{1}{G_{2}} \sum_{i \neq a, i \in \Omega} \alpha_{i b} P_{t o t}}\right)
$$

Note that the above algorithm is not optimal, but is designed to demonstrate a simple distributed implementation. The random transmitter selection in the above algorithm is similar to the technique used in the Grossglauser-Tse relay algorithm of [13]. However, rather than transmitting to the nearest receiver, the algorithm chooses the receiver to improve the backlog-rate metric given in (22). It can be shown to achieve a stability region that contains the stability region of the relay algorithm when transmit probability of the relay algorithm is set to $1 / 2$ (which is a near optimal operating point for the relay simulations considered in [13]). In particular, in a fully mobile environment, it achieves a capacity which does not vanish as the number of nodes is increased.

\section{Vi. Implementation for Mobile Ad-Hoc Networks}

Here we apply the Enhanced DRPC policy to an an ad-hoc network with mobility and inter-channel interference. Consider a square network with $N$ users, with user locations discretized to a $K \times K$ grid. The stochastic channel process $\underline{S}(t)$ is characterized by the following stochastic model of user mobility: Every timeslot, users keep their locations with probability $1 / 2$, and with probability $1 / 2$ they move one step in either the North, South, West, or East directions (uniformly distributed over all feasible directions). Each user is power constrained to $P_{t o t}$, is restricted to transmitting to only one other user in a given timeslot, and cannot transmit if it is receiving. Power radiates omnidirectionally, and signal attenuation between two nodes $a$ and $b$ is determined by the $4^{\text {th }}$ power of the distance between them (as in [7]), so that fading coefficients are given by:

$$
\alpha_{a b}= \begin{cases}1 /\left[\left(\left(x_{a}-x_{b}\right)^{2}+\left(y_{a}-y_{b}\right)^{2}\right)^{2}+1\right] & \text { if } a \neq b \\ \infty & \text { if } a=b\end{cases}
$$

where $\left(x_{a}, y_{a}\right),\left(x_{b}, y_{b}\right)$ represent user locations within the network. Note that the extra ' +1 ' term in the denominator is inserted to model the reality that attenuation factors $\alpha_{a b}$ are kept below 1 (so that signal power at the receiver is never more than the corresponding power used at the transmitter). The $\alpha_{a a}$ values are set to infinity to enforce the constraint that transmitting nodes cannot receive.

Multi-user interference is modeled similarly to the ratepower curve given in (1). However, rather than use the $\log (1+$ $S I R$ ) function, we use a rate curve determined by four different QAM modulation schemes designed for error probabilities less than $10^{-6}$. The rate function is thus:

$$
\mu_{a b}(\underline{P}, \underline{\alpha})=\Phi\left(S I R_{a b}(\underline{P}, \underline{\alpha})\right)
$$

where $\Phi()$ is a piecewise constant function of the signal-tointerference ratio defined by the coding schemes given in Fig. 3. We take the $S I R_{a b}()$ function to be the same as that used in eq. (1), where we assume the CDMA gain parameters are $G_{1}=G_{2}=1$.

We consider the Enhanced DRPC algorithm with $\theta_{i}^{c}=1$, $V_{i}^{c}=1$ for all $i \neq c$, and $V_{i}^{i}=0$, and assume the power/noise coefficient is normalized to $P_{t o t} / N_{b}=20 \Delta^{2}$, where $\Delta$ is the minimum distance between signal points in the QAM modulation scheme. The algorithm is approximated using the distributed implementation described in the previous section, where each node transmits using full power with probability $1 / 2$. A centralized implementation is also considered, where the optimization problem (22) is implemented using a steepest ascent search on the piecewise linear relaxation of the $\Phi(S I R)$ curve (see Fig. 3). The resulting data rates are then 'floored' according the threshold levels of the piecewise constant curve $\Phi(S I R)$. Note that the relaxed problem remains non-linear and non-convex (because SIR is non-convex in the power variables, see (1)), and hence the result of the steepest ascent search may be sub-optimal.

We simulate the centralized and decentralized implementations of DRPC and compare to the performance offered by the 2-hop relay algorithm presented in [13]. The relay algorithm restricts routes to 2-hop paths, and hence relies on rapid user mobility for delivering data. We set the sender density parameter of the relay algorithm to $1 / 2$. Note that the relay algorithm was developed to demonstrate non-vanishing capacity for large networks, and was not designed to maximize throughput or achieve low delay. Thus, it is not completely fair to compare performance with the DRPC algorithms. However, the comparison illustrates the capacity gains and delay reductions that can be achieved in this mobile ad-hoc network setting.

The relay algorithm was designed for nodes to transmit data at a fixed rate, attainable whenever the SIR for a given wireless link exceeds a threshold value. For scheduling purposes, we

\begin{tabular}{|c|c|c|}
\hline modulation & bits/symbol & SIR/symbol \\
\hline 2 PAM & 1 & $0.25 \Delta^{2}$ \\
\hline 4 QAM & 2 & $0.50 \Delta^{2}$ \\
\hline 16 QAM & 4 & $1.25 \Delta^{2}$ \\
\hline 64 QAM & 6 & $5.25 \Delta^{2}$ \\
\hline
\end{tabular}

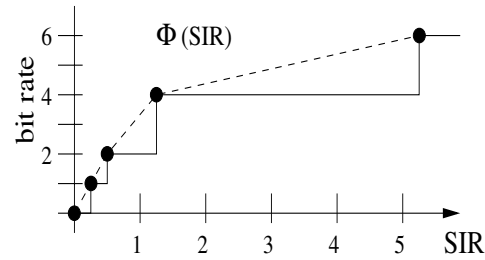

Fig. 3. A piecewise constant rate curve for the 4 modulation schemes described in the table. Scaled power requirements are shown, where $\Delta$ represents the minimum distance between signal points. 
use the 4 bits/symbol threshold corresponding to the 16 QAM scheme of Fig. 3. However, in order to make a fair comparison, once scheduling decisions have been made we allow the relay algorithm to transmit at rates given by the full $\Phi(S I R)$ curve.

Here we consider a small network with 10 users communicating on a $5 \times 5$ square region. Following the scenario of [13], we assume user $i$ desires communication with only one other user (namely, user $(i+1) \bmod N$ ). Unit length packets arrive according to Poisson processes, where 9 of the users receive data at rate $\lambda_{1}$, and the remaining user receives data at rate $\lambda_{2}$. In Fig. 4 we plot the average network delay from simulation of the three algorithms when the rates $\left(\lambda_{1}, \lambda_{2}\right)$ are linearly scaled upwards to the values $(.625,3.125)$. From the figure, we see that the centralized DRPC algorithm provides stability and bounded delays at more than four times the data rates of the relay algorithm, and more than twice the data rate of the decentralized DRPC algorithm. Note that the relay algorithm offers the best delay performance in the low-rate regime. From the graph, it is apparent that this occurs only when the average occupancy $\bar{U}$ in each node of the system is less than 10 packets (note that performance is plotted on a log scale). Performance of the DRPC algorithms in this low rate regime can be improved by using parameter values $V_{i}^{(i+1)} \bmod N=2, V_{i}^{i}=0, V_{i}^{c}=1$ for $c \neq i,(i+1) \bmod N$, which biases packets to 2-hop paths, although we omit this comparison for brevity. We further note that the relay algorithm relies on full and homogeneous mobility of all users, while the DRPC algorithms have no such requirement and can be used for heterogeneous networks with limited mobility.

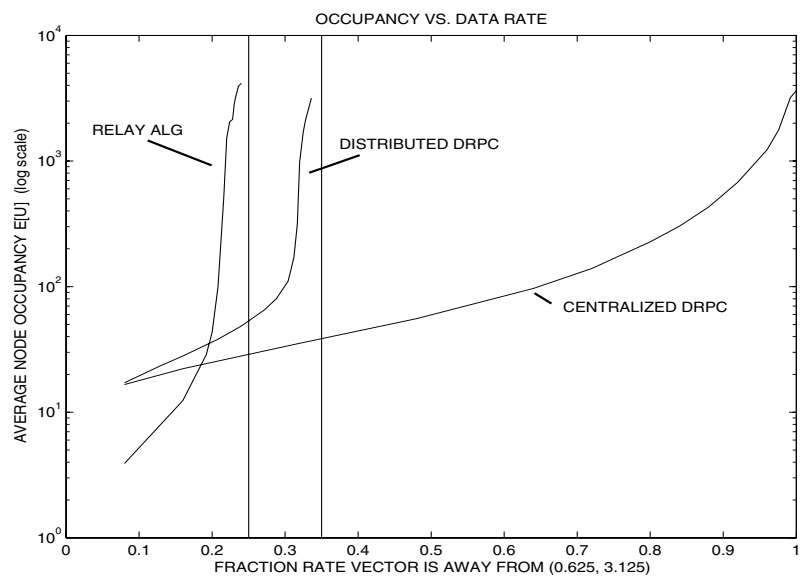

Fig. 4. Simulation results for the DRPC algorithm and the relay algorithm as rates are increased towards $\left(\lambda_{1}, \lambda_{2}\right)=(0.625,3.125)$.

\section{CONCLUSIONS}

We have formulated a general power allocation problem for a multi-node wireless network with time varying channels and adaptive transmission rates. The network capacity region was established, and a Dynamic Routing and Power Control (DRPC) algorithm was developed and shown to stabilize the network whenever the arrival rate matrix is within the capacity region. Such stability holds for general arrival and channel processes, even if these processes are unknown to the network controller. A delay bound was provided for the case when arrivals and channel states are iid from slot to slot.
The algorithm involves solving a constrained optimization problem each timeslot, where queue backlogs and channel conditions occur as parameters in the optimization. Centralized and decentralized approximations were considered for a mobile adhoc network. Algorithms which make more effort to maximize the optimization metric by exchanging backlog and channel information were shown to have significant performance advantages, as illustrated by the example simulations. We believe that such dynamic strategies will be useful in the future for enabling high data rates and low delays.

\section{APPENDIX}

Necessary Condition for Network Stability (From Theorem 1): Here we establish that $\left(\lambda_{i j}\right) \in \Lambda$ is a necessary condition for stability in a wireless network. The proof uses the following preliminary lemma:

Lemma 4. (Compact Set Integration) Suppose an instantaneous rate matrix $\underline{\mu}(t)$ is integrable and lies within a compact set $\Omega$ for all time. Then $\frac{1}{t} \int_{0}^{t} \underline{\mu}(\tau) d \tau$ lies within the convex hull of $\Omega$.

Proof: The integral can be expressed as a limit of a summation with $K$ terms:

$$
\frac{1}{t} \int_{0}^{t} \underline{\mu}(\tau) d \tau=\lim _{K \rightarrow \infty} \frac{1}{K} \sum_{k=1}^{K} \underline{\mu}(k t / K)
$$

For each finite value $K$, the summation represents a convex combination of points in $\Omega$, and hence is in the convex hull of $\Omega$. As $K$ tends to infinity, we obtain a sequence of points in the convex hull of $\Omega$ converging to the integral, which is thus a limit point of the set. Because $\Omega$ is compact, the convex hull is compact and hence contains its limit points.

Theorem 1a. (Necessary Condition for Stability) The condition $\left(\lambda_{i c}\right) \in \Lambda$ is necessary for network stability.

Proof: Consider a system with ergodic inputs with rates $\left(\lambda_{i c}\right)$, and let process $X_{i}^{(c)}(t)$ represent the total bits that have entered the network during the interval $[0, t]$ for each commodity $c \in\{1, \ldots, N\}$. Suppose the system is stabilizable by some routing and power control policy, perhaps one which bases decisions upon complete knowledge of future arrivals and channel states. Note that although the policy stabilizes the system, the power allocations $\underline{P}(t)$ are not necessarily ergodic, nor are the internal bit streams produced by routing decisions. Let $U_{i}^{(c)}(t)$ represent the resulting unfinished work function for commodity $c$ in node $i$ under this stabilizing policy. Further, let $F_{a b}^{(c)}(t)$ represent the total number of bits from commodity $c$ transmitted over the $(a, b)$ link during the interval $[0, t]$. We have for all time:

$$
\begin{aligned}
& F_{a b}^{(c)}(t) \geq 0 \quad \forall a, b, c \\
& X_{i}^{(c)}(t)-U_{i}^{(c)}(t)=\sum_{b} F_{i b}^{(c)}(t)-\sum_{a} F_{a i}^{(c)}(t) \quad \forall i \neq c \\
& \sum_{k} X_{k}^{(c)}(t)-\sum_{k} U_{k}^{(c)}(t)=\sum_{a} F_{a c}^{(c)} \quad \forall c \\
& \sum_{c} F_{a b}^{(c)}(t) \leq \int_{0}^{t} \mu_{a b}(\underline{P}(\tau), \underline{S}(\tau)) d \tau \quad \forall(a, b)
\end{aligned}
$$

where (35) follows because the unfinished work in any node is equal to the difference between the total bits that have arrived and departed, and (36) follows because the number of 
commodity $c$ bits successfully delivered to node $c$ is equal to the total commodity $c$ bits that arrived from exogenous sources minus those bits which are still inside the network. Inequality (37) holds because the total bits transferred over any link $(a, b)$ is less than or equal to the offered transmission rate integrated over the time interval $[0, t]$.

Let $T_{\underline{S}}(t)$ represent the subintervals of $[0, t]$ during which the channel is in state $\underline{S}$, and let $\left\|T_{\underline{S}}(t)\right\|$ denote the total length of these subintervals. Fix an arbitrarily small value $\epsilon>0$. Because the channel process $\underline{S}(t)$ is ergodic on a finite state space, and because there are a finite number of ergodic input streams $X_{c}(t)$, when measured over any sufficiently large interval $[0, t]$ the time average fraction of time in each channel state and the empirical average data rate of all inputs are simultaneously within $\epsilon$ of their limiting values. Furthermore, by the Network Stability Lemma (Lemma 1), there must exist some finite value $M$ such that the unfinished work in all queues is simultaneously less than $M$ at arbitrarily large times. Hence, there exists a time $\tilde{t}$ such that:

$$
\begin{gathered}
U_{i}^{(c)}(\tilde{t}) \leq M \text { for all nodes } i \text { and commodities } c \\
\qquad \frac{M}{\tilde{t}} \leq \epsilon \\
\left|\frac{X_{i c}(\tilde{t})}{\tilde{t}}-\lambda_{i c}\right| \leq \epsilon \\
\frac{\left\|T_{S}(\tilde{t})\right\|}{\tilde{t}} \leq \pi_{\underline{S}}+\epsilon
\end{gathered}
$$

Now define variables $f_{a b}^{(c)} \triangleq F_{a b}^{(c)}(\tilde{t}) / \tilde{t}$. Applying inequality (37) at time $\tilde{t}$, dividing by $\tilde{t}$, and considering entrywise matrix inequalities, we have:

$$
\begin{aligned}
\left(\sum_{c} f_{a b}^{(c)}\right) & \leq\left(\frac{1}{\tilde{t}} \int_{0}^{\tilde{t}} \mu_{a b}(\underline{P}(\tau), \underline{S}(\tau)) d \tau\right) \\
& =\sum_{\underline{S}} \frac{\left\|T_{\underline{S}}(\tilde{t})\right\|}{\tilde{t}} \frac{1}{\left\|T_{\underline{S}}(\tilde{t})\right\|}\left(\int_{\tau \in T_{\underline{S}}(\tilde{t})} \mu_{a b}(\underline{P}(\tau), \underline{S}) d \tau\right) \\
& \leq \sum_{\underline{S}} \frac{\left\|T_{\underline{S}}(\tilde{t})\right\|}{\tilde{t}}\left(\mu \frac{S}{a b}\right)
\end{aligned}
$$

where the matrices $\left(\mu \frac{S}{a b}\right)$ in (44) are elements of Convex_Hull $\{\underline{\mu}(\underline{P}, \underline{S}) \mid \underline{P} \in \Pi\}$. Such values are guaranteed to exist and satisfy the inequality by the Compact Set Integration Lemma (Lemma 4). Specifically, this lemma can be applied using the time average integral in (43) with the compact set of matrices $\{\underline{\gamma} \geq 0 \mid \underline{\gamma} \leq \underline{R}$ for some $\underline{R} \in$ Convex_Hull $\{\mu(\underline{P}, \underline{S}) \mid \underline{P} \in \bar{\Pi}\}\}$. (It is straightforward to show this set is compact for each $\underline{S}$, using compactness of $\Pi$ and upper-semicontinuity of $\mu(\underline{P}, \underline{S})$ [22]). Using (41) in (44), we find:

$$
\left(\sum_{c} f_{a b}^{(c)}\right) \leq \sum_{\underline{S}} \pi_{\underline{S}}\left(\mu_{a b}^{\underline{S}}\right)+\epsilon\left(\mu_{a b}^{\max }\right) \operatorname{Card}\{\underline{S}\}
$$

where $\operatorname{Card}\{\underline{S}\}$ represents the number of channel states $\underline{S}$, and $\mu_{a b}^{\max }$ represents the maximum transmission rate of the $(a, b)$ link over all channel states and power levels $\underline{P} \in \Pi$. Hence, the right hand side of inequality (45) is arbitrarily close to a point in $\Gamma$ (c.f. (7)). Furthermore, (38)-(40) can be used in (35) and (36) to show that the $f_{a b}^{(c)}$ values are arbitrarily close to satisfying the multi-commodity flow conditions (5), (6). Thus, the input rates $\left(\lambda_{i c}\right)$ are arbitrarily close to a point in the capacity region $\Lambda$.
Because $\Lambda$ is compact and hence contains its limit points, it follows that $\left(\lambda_{i c}\right) \in \Lambda$.

\section{REFERENCES}

[1] M.J. Neely, E. Modiano, and C.E.Rohrs, "Power and Server Allocation in a Multi-Beam Satellite with Time Varying Channels," IEEE Proceedings of INFOCOM 2002.

[2] M.J. Neely, E. Modiano, and C.E. Rohrs, "Dynamic Routing to Parallel Time-Varying Queues with Application to Satellite and Wireless Networks," Proceedings of the Conference on Information Sciences and Systems, Princeton, March 2002.

[3] B. Prabhakar, Elif Uysal Biyikoglu, and A. El Gamal, "Energy-Efficient Transmission Over a Wireless Link via Lazy Packet Scheduling," IEEE Proceedings of INFOCOM 2001.

[4] A. Fu, E. Modiano, J. Tsitsiklis, "Optimal Energy Allocation for DelayConstrained Data Transmission over a Time-Varying Channel," IEEE Proceedings of INFOCOM 2003.

[5] L. Xiao, M. Johansson, S. Boyd, "Simultaneous Routing and Resource Allocation for Wireless Networks," Proceedings of the 39th Annual Allerton Conference on Communication, Control, and Computing, Oct. 2001.

[6] D. Julian, M. Chiang, D. O'Neill, S. Boyd, "QoS and Fairness Constrained Convex Optimization of Resource Allocation for Wireless Cellular and Ad Hoc Networks," IEEE Proceedings of INFOCOM 2002.

[7] T. ElBatt, A. Ephremides, "Joint Scheduling and Power Control for Wireless Ad-hoc Networks," IEEE Proceedings of INFOCOM 2002.

[8] P. Marbach, "Priority Service and Max-Min Fairness," IEEE Proceedings of INFOCOM 2002.

[9] F.P. Kelly, A.Maulloo, D. Tan, "Rate Control for Communication Networks: Shadow Prices, Proportional Fairness, and Stability," Journal of the Operational Research Society 49, p.237-252, 1998.

[10] P. Marbach and R. Berry, "Downlink Resource Allocation and Pricing for Wireless Networks," IEEE Proceedings of INFOCOM 2002.

[11] P. Gupta and P.R. Kumar, "Critical Power for Asymptotic Connectivity in Wireless Networks," IEEE Conf. on Decision and Control, 1998.

[12] P. Gupta and P.R. Kumar, "The Capacity of Wireless Networks," IEEE Transactions on Information Theory, 46:388-404, March 2000.

[13] M. Grossglauser and D. Tse, "Mobility Increases the Capacity of Ad-hoc Wireless Networks," IEEE Proceedings of INFOCOM, 2001.

[14] L. Tassiulas and A. Ephremides, "Stability Properties of Constrained Queueing Systems and Scheduling Policies for Maximum Throughput in Multihop Radio Networks," IEEE Transactions on Automatic Control, Vol. 37, no. 12, Dec. 1992

[15] L. Tassiulas, "Scheduling and performance limits of networks with constantly changing topology," IEEE Trans. on Information Theory, May 1997.

[16] N. McKeown, V. Anantharam, and J. Walrand, "Achieving $100 \%$ throughput in an input-queued switch," IEEE Proceedings of INFOCOM, 1996, pp. 296-302.

[17] E. Leonardi, M. Mellia, F. Neri, and M. Ajmone Marson, "Bounds on Average Delays and Queue Size Averages and Variances in Input-Queued Cell-Based Switches," IEEE Proceedings of INFOCOM 2001, vol 2.

[18] P.R.Kumar, S.P.Meyn, "Stability of Queueing Networks and Scheduling Policies," IEEE Transactions on Automatic Control, vol.40,.n.2, Feb. 1995, pp.251-260.

[19] M. J. Neely, E. Modiano, and C.E. Rohrs, "Tradeoffs in Delay Guarantees and Computation Complexity for $N \times N$ Packet Switches," Proceedings of the Conference on Information Sciences and Systems, Princeton, March 2002.

[20] J.P. Choi and V.W.S. Chan, "Predicting and Adapting Satellite Channels with Weather-Induced Impairments" IEEE Trans. on Aerospace and Electronic Syst., July 2002.

[21] Soren Asmussen, Applied Probability and Queues. New York: John Wiley, 1987.

[22] D.P. Bertsekas, A. Nedic, and A.E. Ozdaglar, Convex Analysis and Optimization. To be published: Athena Scientific, Feb. 2003.

[23] M.J. Neely, Dynamic Power Allocation and Routing in Satellite and Wireless Networks with Time Varying Channels. Ph.D. Dissertation, Massachusetts Institute of Technology, LIDS 2003.

[24] R.G. Gallager, Discrete Stochastic Processes. Kluwer Publishers: Boston 1995.

[25] A. El Gamal, C. Nair, B. Prabhakar, E. Uysal-Biyikoglu,S. Zahedi, "Energy-efficient Scheduling of Packet Transmissions over Wireless Networks," IEEE Proceedings of INFOCOM 2002.

[26] S. Shakkottai, R. Srikant, A. Stolyar, "Pathwise Optimality and State Space Collapse for the Exponential Rule," IEEE International Symposium on Informatin Theory, 2002.

[27] S. Toumpis and A. Goldsmith, "Some Capacity Results for Ad-Hoc Networks," 38th Annual Allerton Conference Proceedings, 2000. 